NOTE TECHNIQUE No 0.5

\title{
SUR L'UTILISATION DU FORMOL EN THERAPEUTIQUE PISCICOLE
}

\author{
établie par M. J.-P. GERARD, Docteur Vétérinaire \\ Laboratoire d'Ichtyopathologie de I'INRA \\ 78850 THIVERVAL-GRIGNON
}

\section{Présentation :}

Le «formol du commerce "utilisé pour le traitement des poissons est le produit obtenu par la dissolution d'un gaz "l'aldéhyde formique " dans l'eau. Cette solution est généralement incolore à la température ambiante, la présence d'un dépôt blanchâtre n'altère en rien la qualité du produit. La solubilité de ce gaz dans l'eau n'est que de 30 à $40 \%$.

Toutes les posologies que nous indiquons sont uniquement ètablies pour l'emploi des solutions commerciales et non de "l'aldéhyde formique ".

Cette précision est très importante car elle entraine une variation de un à trois environ et les poissons ne supportent pas ce surdosage.

\section{Indications :}

Le formol est surtout actif contre les ectoparasites (costia, trichodina) de faible taille et dont la mise en évidence ne peut être effectuée qu'à l'aide d'un appareil optique. Il sera employé également pour traiter les alevins afin d'éviter le développement de ces parasites.

Nota : Alors que l'aldéhyde formique est un excelient désinfectant, le formol ne sera jamais utilisé pour la désinfection, il faut lui préférer soit l'eau de javel, soit les ammoniums quaternaires.

\section{Toxicité :}

Aux doses therrapeutiques indiquees, le formol n'est pas toxique pour les poissons à condition de respecter la durée et la dose pour un bain effectué à une température déterminée de l'eau.

\section{Posologie :}

Le formol est utilisé aux doses suivantes :

- $250 \mathrm{ppm}^{*}$ dans de l'eau dont la température est inférieure à $10^{\circ}$

- $200 \mathrm{ppm}$ si la température est comprise entre 100 et $15^{\circ}$

- $160 \mathrm{ppm}$ si la température de l'eau est supérieure à 150 .

\footnotetext{
- ppm : partie par million, soit : 1 gramme par mètre cube ou milligramme par litre
} 


\section{Mode d'emploi :}

Les traitements sont surtout effectués dans les auges d'alevinage et nous conseillons de procéder de la façon suivante :

1) Les poissons à traiter seront à jeun de 12 heures.

2) Evaluer le volume d'eau à traiter et calculer la quantité de formol nécessaire ( $1 / 4$ de litre de formol par mètre cube d'eau à traiter).

3) Prédiluer cette quantité dans un récipient, par exemple un arrosoir qui contiendra donc une solution mère concentrée.

4) Arrêter l'arrivée d'eau.

5) Répartir la solution concentrée de formol dans le bac d'alevinage puis brasser à l'aide d'un balai propre pour obtenir une solution homogène.

6) Après 15 minutes les $2 / 3$ de l'eau sont vidangés et l'arrivée d'eau rétablie simultanément.

\section{Périodicité des traitements :}

\section{A) Prèventivement :}

- Si le formol est employé seul des traitements périodiques préventifs devront être effectués tous les 10 jours.

Si le traitement au formol est combiné à d'autres traitements, nous proposons le calendrier suivant, en prenant comme jour de départ la fin de la résorption vésiculaire.

Jours après résorption

\section{5}

15

25

32

39

44

50

58

64

70

80

90

\section{Traitements}

Formol 250 ppm, 15 minutes

Formol

Ammoniums quaternaires $2 \mathrm{ppm}$

Formol

Ammonium quaternaire ou Furanace

Formol

Ammonium quaternaire ou Furanace

Formol

Ammonium quaternaire ou Furanace ou Sulfate de cuivre (fiche technique $n^{\circ}$ 1)

Formol

Ammonium quaternaire ou Furanace ou Sulfate de cuivre

Formol

\section{B) Curativement :}

Si aucun traitement préventif n'a été mis en œuvre et qu'une mortalité importante survient par ectoparasitose, 3 traitements à 24 heures d'intervalle pourront être effectués, puis un autre 48 heures après le dernier, puis un tous les dix jours. 